\title{
Graphene on hexagonal lattice substrate: Stress and Pseudo-magnetic field
}

\author{
M. Neek-Amal and F.M. Peeters \\ Department of Physics, University of Antwerpen, \\ Groenenborgerlaan 171, B-2020 Antwerpen, Belgium
}

(Dated: August 13, 2021)

\begin{abstract}
Moiré patterns in the pseudo-magnetic field and in the strain profile of graphene (GE) when put on top of a hexagonal lattice substrate are predicted from elasticity theory. The van der Waals (vdW) interaction between GE and the substrate induces out-of-plane deformations in graphene which results in a strain field, and consequently in a pseudo-magnetic field. When the misorientation angle is about $0.5^{\circ}$ a three-fold symmetric strain field is realized that results in a pseudo-magnetic field very similar to the one proposed by F. Guinea, M. I. Katsnelson, and A. K. Geim [Nat. Phys. 6, 30 (2010)]. Our results show that the periodicity and length of the pseudo-magnetic field can be tuned in GE by changing the misorientation angle and substrate adhesion parameters and a considerable energy gap $(23 \mathrm{meV})$ can be obtained due to out-of-plane deformation of graphene which is in the range of recent experimental measurements $(20-30 \mathrm{meV})$.
\end{abstract}

Stacking different two dimensional materials with slightly different lattice structures on top of each other results in a new superlattice structure which is called Moiré pattern. The van der Waals (vdW) interaction between different 2D-crystals such as graphene (GE), hexagonal boron nitride (h-BN), and molybdenum disulfide $\left(\mathrm{MOS}_{2}\right)$ results in a multilayer heterostructure [1]. The resulting hexagonal Moiré pattern in graphene on top of other hexagonal lattice substrates affects the electromechanical properties of graphene. For example, hexagonal boron nitride has turned out to be an ideal dielectric substrate which is atomically flat and improves graphene's mobility by more than two orders of magnitude [2, 3]. The $\mathrm{B}-\mathrm{N}$ bond length is close to that of $\mathrm{C}-\mathrm{C}$ with only a very small (1.6\%-2\%) lattice mismatch [4, 5] which results in the appearance of a Moiré pattern (MP) when GE is put on top of BN. It was found that GE flakes can align with the underlying h-BN lattice within an error of less than $0.05^{\circ}[4,6]$. Ab-initio and semi-empirical van der Waals studies showed that the interaction between GE flakes and the h-BN substrate is similar to that of a GE-GE stacked structure [7]. On the other hand the different electronegativity of $\mathrm{B}, \mathrm{N}$ and $\mathrm{C}$ atoms leads to a nonuniform attractive force distribution over GE.

Non-uniform strain in GE results in a pseudo-magnetic field and consequently results in the opening of an energy gap [8, 9]. Earlier density functional theory calculations assumed lattice matching between GE and h-BN which induces in-plane strain and opens a gap in GE's spectrum of 50-60 meV 7]. But recent experiments found a gap in the range of $20-30 \mathrm{meV}$ [10, 11]. In this letter, we first develop a general theory for GE over a hexagonal lattice substrate and show that the induced strain has triangular symmetry resulting in interesting pseudo-magnetic field patterns which vary with the misorientation angle. Then, as an example, we concentrate on the h-BN-lattice induced deformation of the GE lattice using atomistic simulations and compare with our analytic results. Using experimental height deformation [12] as an input in our analytic theory we found that the pseudo-magnetic field modulation amplitude can be of order 1 Tesla for misori-
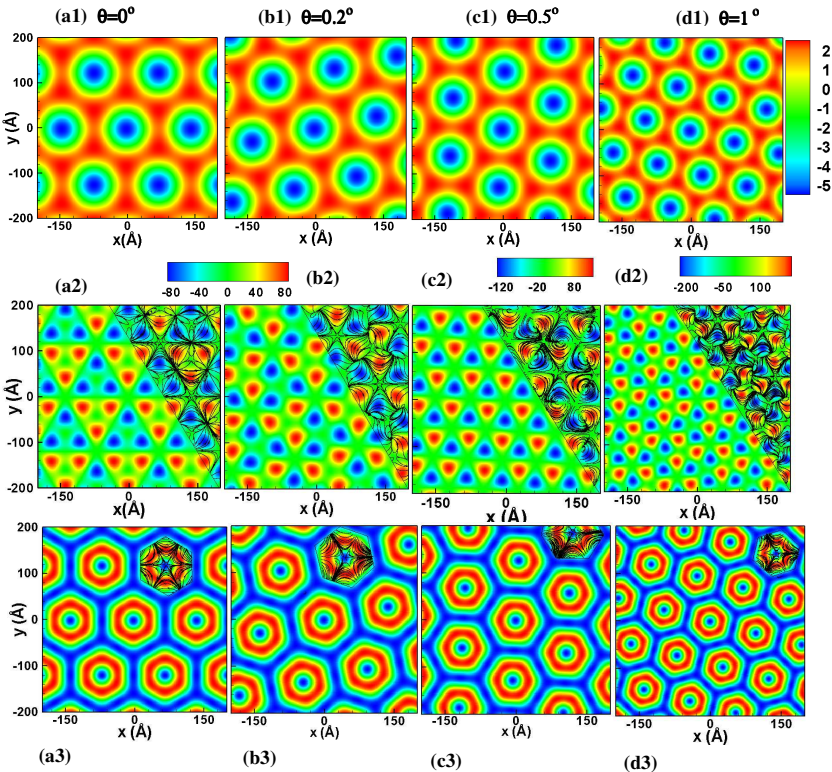

FIG. 1: (a1-c1) Height deformations $\left(\Delta h / h_{0}\right)$ of GE over hBN sheet, i.e. Eq. (1), for different misorientation angle with lattice mismatch $1.7 \%$. (a2-c2) The corresponding induced magnetic field (per $h_{0}^{2}$ ), Eq. (5). Typical streamlines of gauge field vector are shown in the corner of each panel(a3-c3). The strain tensor eigenvalue, i.e. $\epsilon_{+}$(which is equivalent to the absolute value of the gauge field).

entation less than $1^{\circ}$. The latter results in an energy gap of about $23 \mathrm{meV}$. The model. The mismatch between the honeycomb lattice structures of GE and a hexagonal lattice (e.g. h-BN) leads to long wavelength Moiré patterns. For a given lattice mismatch and misorientation between GE and the substrate lattice we expect that the GE sheet is attracted to the h-BN substrate (the adhesion energy for $\mathrm{GE} / \mathrm{h}-\mathrm{BN}$ is about $30-50 \mathrm{meV}$ per atom [7, 13]). For a lattice mismatch $\delta$ and misorientation angle $\theta$ (with the zig-zag (zz) direction along the $\mathrm{x}$-axis) the deformation 
of the lattice due to $\mathrm{vdW}$ adhesion is $\Delta h(x, y)$ which also depends on the $\mathrm{vdW}$ adhesion strength $\varepsilon$. We found that the symmetry of the out-of-plane deformation in the GE lattice is similar to the MP structure [14]. Therefore the Fourier transform (FT) of $\Delta h$ should correspond to six Moiré pattern vectors [4, 13, 15], i.e. $\vec{G}_{m}=\Re_{\phi_{m}} \vec{G}_{0}$ with $m=0,1, . .5$ where $\vec{G}_{0}=\left(\hat{1}-\frac{1}{1+\delta)} \Re_{\theta}\right)(0,2 \kappa)$ with $\kappa=\frac{2 \pi}{3 a_{0}}$ and $\Re_{\phi_{m}}$ (and $\Re_{\theta}$ ) is the rotation matrix about the $z$-axis over an angle $\phi_{m}=\frac{2 \pi m}{6}$ (and $\theta$ ) where $a_{0}=a_{C C}$ is the $\mathrm{C}-\mathrm{C}$ bond length. The height deformation of GE can be generally written as $\Delta h=h_{0} \sum_{m} e^{i \vec{G}_{m} \cdot \vec{r}}$, where $h_{0}$ is the amplitude of the deformation. For $\theta<<1^{\circ}$ we simplify the modulation function as

$$
\frac{\Delta h}{2 h_{0}}=\cos \left[\vec{r} \cdot \overrightarrow{G_{0}}\right]+2 \cos \left[\frac{\vec{r} \cdot \overrightarrow{G_{0}}}{2}\right] \cos \left[\frac{\sqrt{3}}{2} \vec{r} \times \vec{G}_{0}\right] .
$$

The elements of the strain tensor can be found using $\epsilon_{\alpha \beta}=\frac{1}{2} \partial_{\alpha} h \partial_{\beta} h$. The $\mathrm{x}$-component of the strain tensor is a periodic function and shows mirror symmetry along the $\mathrm{zz}$ direction while the y-component shows three fold symmetry with large peaks on the hexagonal sites. The shear component shows two fold symmetry and is three times smaller. Diagonalising the strain tensor gives the principal axis with eigenvalues

$$
\epsilon_{ \pm}=\frac{1}{2}\left[\epsilon_{i i} \pm|\vec{A}|\right]
$$

where $\vec{A}$ is the gauge vector corresponding to the lattice deformation [16]. Surprisingly we found that $\epsilon_{-}=0$ (since $\epsilon_{x x} \epsilon_{y y}=\epsilon_{x y}$ ) and $\epsilon_{+}=|\vec{A}|$ having MP properties. For a two dimensional material we found that the corresponding eigenvectors have an angle

$$
\Phi_{-}=\pi-\tan ^{-1}\left(\frac{\epsilon_{x y}}{\epsilon_{x x}}\right), \Phi_{+}=\pi-\tan ^{-1}\left(\frac{\epsilon_{x y}}{\epsilon_{y y}}\right)
$$

with respect to the zz-direction. Since the eigenvalue $\epsilon_{-}=0$, we conclude that the stress along the corresponding eigenvector results in no lattice deformation.

The low energy electronics of the deformed GE can be obtained from the Dirac equation after inserting the modified hopping parameters from the tight-binding model which are now a function of the atomic positions $t(\mathbf{r})[8]$. Rewriting the Dirac Hamiltonian in the effective mass approximation introduces now the strain induced effective gauge field $\vec{A}=\frac{2 \beta_{0} \hbar}{3 a_{0} e}\left(\epsilon_{x x}-\epsilon_{y y},-2 \epsilon_{x y}\right)$ where $\beta_{0}(\sim 2-3)$ is a constant (the strain due to the out of plane displacements [16]). Using two components of the effective gauge field in the unit $\tau=8 \kappa^{2} \beta_{0} \hbar h_{0}{ }^{2} / 3 a_{0} e$ are we can find the corresponding curvature induced magnetic field perpendicular to the $x-y$ planes in units of $2 \kappa \tau$ given by

$$
\begin{aligned}
B= & 4 \sqrt{3} \theta \omega_{1} \cos \left(\sqrt{3} \kappa \chi_{1}\right) \sin \left(\sqrt{3} \kappa \chi_{1}\right) \cos ^{2}\left(\kappa \chi_{2}\right)-4 \delta \omega_{2}\left[\sin \left(\kappa \chi_{2}\right) \cos ^{2}\left(\sqrt{3} \kappa \chi_{1}\right)+\sin \left(2 \kappa \chi_{2}\right) \cos \left(\sqrt{3} \kappa \chi_{1}\right)+\right. \\
& \left.\left.-3 \sin \left(\kappa \chi_{2}\right) \sin ^{2}\left(\sqrt{3} \kappa \chi_{1}\right)\right] \cos \left(\kappa \chi_{2}\right)+4 \sqrt{(3}\right) \theta \omega_{1} \sin \left(\kappa \chi_{2}\right)\left[\cos \left(\sqrt{3} \kappa \chi_{1}\right) \sin \left(\kappa \chi_{2}\right)+\sin \left(2 \kappa \chi_{2}\right)\right] \sin \left(\sqrt{3} \kappa \chi_{1}\right)+ \\
& \cos \left(2 \kappa \chi_{2}\right)\left[\delta \omega_{2} \cos \left(\sqrt{3} \kappa \chi_{1}\right) \sin \left(\kappa \chi_{2}\right)+\delta \omega_{2} \sin \left(2 \kappa \chi_{2}\right)-4 \sqrt{3} \theta \omega_{1} \cos \left(\kappa \chi_{2}\right) \sin \left(\sqrt{3} \kappa \chi_{1}\right)\right]
\end{aligned}
$$

where $2 \kappa \chi_{1}=\vec{r} \times \overrightarrow{G_{0}}, 2 \kappa \chi_{2}=\vec{r} \cdot \overrightarrow{G_{0}} \omega_{1}=3 \delta^{2}-\theta^{2}$ and $\omega_{2}=3 \theta^{2}-\delta^{2}$. The corresponding stress tensor is given by $\sigma_{i j}=\lambda \delta_{i j} \epsilon_{i i}+2 \mu \epsilon_{i j}$, where $\lambda$ and $\mu$ are the Lamé parameters that determine the stiffness of the material. It is interesting to note that the gauge field is proportional to the main element of the stress tensor, i.e. $\sigma_{+}=\sigma_{i i}=2 K \epsilon_{+}$with $K=12.3 \mathrm{eV} \AA^{-2}$ being the $2 \mathrm{D}$ bulk modulus of GE [17]. However, to find the energy levels and localized states in the $\mathrm{K}$ valley one can solve the following equation for the eigen-energy $\mathrm{E}$ :

$$
v_{F}^{2}\left[\vec{\Pi}^{2} \pm i e(\vec{p} \times \vec{A})\right] \psi_{K_{ \pm}}=E^{2} \psi_{K_{ \pm}}
$$

where $v_{F}$ is the Fermi velocity, \pm refer to the $\mathrm{A}$ and B-sites in the GE lattice and $\vec{\Pi}=\vec{p}+e \vec{A}$.

In Fig. 1 we collect all the results for the height deformations (labeled by 1), pseudo-magnetic field (labeled by 2 in units of $B_{0}=h_{0}^{2} T \AA^{-2}$ ), the $\epsilon_{+}$in arbitrary units (equivalently, the absolute value of gauge field labeled by 3 ) for different misorientation angles, $\theta=0^{\circ}, 0.1^{\circ}, 0.5^{\circ}$ and $1^{\circ}$ from left to right, respectively. The MP structure appears in $\Delta h$ and $\epsilon_{+}$panels with smaller period for larger $\theta$. Notice that the main MP hexagon in the central part of a1 (a3) for $\Delta h\left(\epsilon_{+}\right)$is rotated by an angle $\varphi=\tan ^{-1} \frac{\sin (\theta)}{\cos (\theta)+\delta-1}$ clockwise and is scaled by $L=a_{0}\left[2(1-\delta)(1-\cos (\theta))+\delta^{2}\right]^{-1 / 2}$. It is seen that for $\theta=0.5^{\circ}(\mathrm{c} 1)$ the height profile is rotated over $\pi / 2$ with respect to the zero angle (a1) result. Notice that the symmetry of the pseudo-magnetic field profiles vary with $\theta$. The $\theta$ angle which reproduces the pseudo-magnetic field results of Ref. [9] is $\theta=0.5^{\circ}$ (i.e. triaxially stressed $\mathrm{GE})$. The latter is related to the angle $\varphi\left(\theta=0.5^{\circ}\right) \simeq \pi / 6$. Notice that the magnetic field profile for $\theta=0^{\circ}$ is completely different from what one expects intuitively, i.e. there is no hexagonal symmetry as in the $0.5^{\circ}$ case. Here, the obtained pseudo-magnetic field is only due to out-ofplane deformation. If in-plane triaxial stress is applied it will increase the pseudo-magnetic field further [8, 9] The change in the pseudo-magnetic field with $\theta$ is a very 
promising method to tune the electronic gap with the lattice misorientation. The profiles in $\epsilon_{+}$do not have a sinusoidal shape along particular directions, e.g. in c3 along $(1, \sqrt{3})$. The blue hexagonal patterns in a3-d3 are the minimum value of the strained part in GE which are connected to each other with the MP structure. However the highest strained regions (red hexagons) show smaller hexagonal patterns. Between these two regions (bluebigger hexagon and red-smaller hexagons) there are moderate green hexagons. The latter effect is closely related to the recent PeakForce tapping atomic force microscopy measurement of the mechanical properties of GE/h-BN where the tip is sensitive to the strain distribution in graphene [10]. The corresponding cross sections of panel a 2 are shown by blue-solid lines for the pseudo-magnetic field in Fig. 2(a). In Fig. 2(b) we show cross sections of $\Delta h$ and $\epsilon_{+}$along the ac direction for $\theta=0$ (a1, a3 blue solid lines) and $0.5^{\circ}$ (c1, c3 red dashed lines).

In Figs. 2(c,d) we depict the solution of Eq. (5), i.e. the probability density of wave function, at the A- and Blattice $\left(\psi_{ \pm}\right)$for low energy $\mathrm{E}=0.3 \mathrm{eV}$ where in (c) $\theta=0^{\circ}$ and in (d) $\theta=0.5^{\circ}$. The hexagons indicate the corresponding MP. Notice that the probability densities are completely different even for small misorientation angle.

Our analytical MP scale free results presented in Figs. 1(a1-c1) are in good agreement with recent experiments on epitaxial grown graphene on h-BN [18]. From the experimental measured amplitude $\Delta h \simeq$ $40 \mathrm{pm}$ and using $h_{0} \sim \Delta h / 5.0$, the pseudo-magnetic field in Fig. 1(a2) varies in the $[-0.5,0.5] \mathrm{T}$ range (i.e. $B_{0}=6.4 \mathrm{mT}$ ). We estimated the energy gap using the classical approximation for relativistic electrons in the presence of magnetic field where the Landau level energy sequence can be written as

$$
E_{N}= \pm \sqrt{2 e \hbar v_{F}^{2} B N} \approx \pm 400 k_{B} \sqrt{B N}
$$

The energy gap can be approximated as $\Delta \simeq 33.3 \mathrm{meV}$ for $\mathrm{B}=1 \mathrm{~T}$. The experimentally observed strain distribution in graphene over h-BN was found to be different for commensurate and incommensurate states [10] which can be equivalent to the transition from a non-uniform to an uniform strain distribution in graphene. The nonuniform strain in graphene results in an opening of a gap in some graphene over h-BN [9], e.g. atomic force microscopy measurement predicts a gap of $\sim 30 \mathrm{meV}$ [10] and $20 \mathrm{meV}$ [11]. The experimentally measured amplitude $\Delta h \simeq 40 \mathrm{pm}$ results in $\mathrm{B} \sim 0.5 \mathrm{~T}$ and by using Eq. (6) in our model we find $\Delta \approx 23 \mathrm{meV}$ which is comparable with available experimental results.

Finally, in order to determine $h_{0}$ and find the scale $B_{0}$ in the previous analysis and have an independent check for the above theory we use a Lennard-Jones potential containing both the short range repulsive and long range attractive nature of the interaction between two particles i.e. $\left.u(r)=4 \varepsilon\left[(\sigma / r)^{12}-(\sigma / r)^{6}\right)\right]$, where $\mathrm{r}$ is the distance between two atoms, $\varepsilon$ is the depth of the

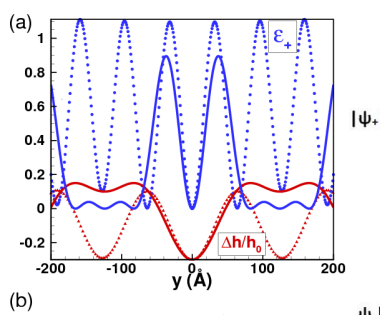

(b)
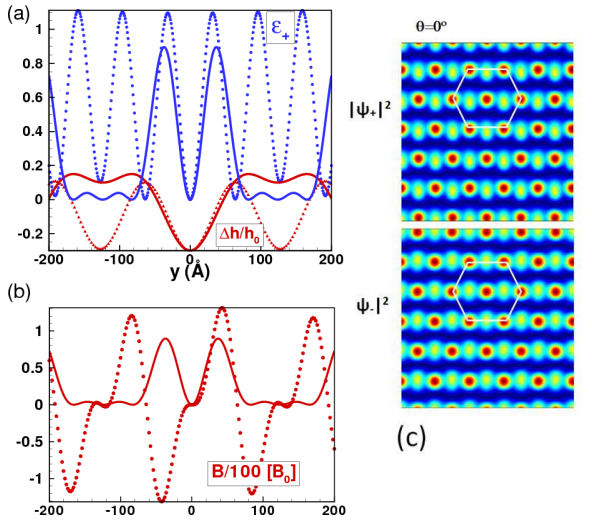

(c)

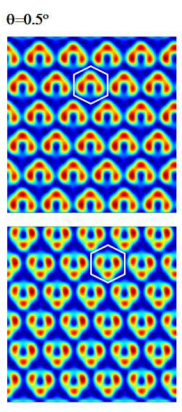

(d)

FIG. 2: (a) The red lines are two cross sections along the ac direction, i.e. $x=0$, from a2 (solid-line $\theta=0^{\circ}$ ) and c2 (dashedline $\theta=0.5^{\circ}$ ) panels (for pseudo-magnetic field) in Fig. 1] (b) The red lines are two cross sections along ac direction, i.e. $x=0$, from a1 (solid-line $\theta=0^{\circ}$ ) and c1 (dashed-line $\theta=0.5^{\circ}$ ) panels in Fig. 11 for height deformation. The corresponding strain eigenvalues (and absolute value of gauge vector) are shown by blue color, i.e. a3 (solid-line $\theta=0^{\circ}$ ) and c3 (dashedline $\theta=0.5^{\circ}$ ) panels. (c,d) The solution of continuum Dirac equation (Eq. (5)) for GE over a substrate with hexagonal lattice structure, $\psi_{ \pm}$are the wave functions over A- and Bsites for low energy $\mathrm{E}=0.3 \mathrm{eV}$.

potential well, and $\sigma$ is the distance at which the potential becomes zero. To model the interaction between $\mathrm{B}, \mathrm{N}$ and $\mathrm{C}$ atoms, we adjust the LJ parameters using the equations $\varepsilon=\sqrt{\varepsilon_{i} \varepsilon_{j}}$ and $\sigma=\left(\sigma_{i}+\sigma_{j}\right) / 2$ where $i, j$ refer to $\mathrm{B}, \mathrm{N}$ or $\mathrm{C}$ and where $\sigma_{C}=3.369 \AA, \sigma_{B}$ $=3.453 \AA, \sigma_{N}=3.365 \AA$ and $\varepsilon_{C}=2.635 \mathrm{meV}, \varepsilon_{B}=4.16$ $\mathrm{meV}$, and $\varepsilon_{N}=6.281 \mathrm{meV}$ [13]. We relaxed the computational unit cell of GE/h-BN (for $\theta=0^{\circ}$ and $\theta=0.5^{\circ}$ ) using molecular dynamics relaxation with the conjugated gradient algorithm (with periodic boundary condition). The GE sample and h-BN layer consist of $250632 \mathrm{C}$ and $242208 \mathrm{~B}$ and $\mathrm{N}$ atoms, respectively (i.e. $N_{t o t}=492840$ ). After relaxation we found that the average $\mathrm{C}-\mathrm{C}$ bond length in GE is $a_{C C}=1.406 \AA$ and $a_{B N}=1.43 \AA$ in h$\mathrm{BN}$ which results in a lattice mismatch $\left(\delta=\frac{a_{B N}}{a_{C C}}-1\right)$ of $1.7 \%$. In Figs. $3(\mathrm{a}, \mathrm{b})$ we depict height deformations in GE due to the interaction with the h-BN sheet for $\theta=0^{\circ}$ along (a) zigzag direction where $y=0$ and (b) armchair direction where $x=0$. The corresponding symbols are the analytical results given by Eq. (1) (see Fig. 1(a1)). It is seen that the MD relaxed deformation is in good agreement with our analytical theory. However, the scale parameter $h_{0}$ was found to be $1.1 \mathrm{pm}$ for $\theta=0^{\circ}$ and $0.1 \mathrm{pm}$ for $\theta=0.5^{\circ}$ which is one order of magnitude smaller than found experimentally [18]. As was also noticed recently for the graphene/ $\mathrm{Cu}(111)$ system [19] this discrepancy can be traced back to the use of a simple pairwise potential that underestimates $h_{0}$. Possibly the use of the yet unavailable three body potential for $\mathrm{C}-\mathrm{B}$ and $\mathrm{C}-\mathrm{N}$ will 

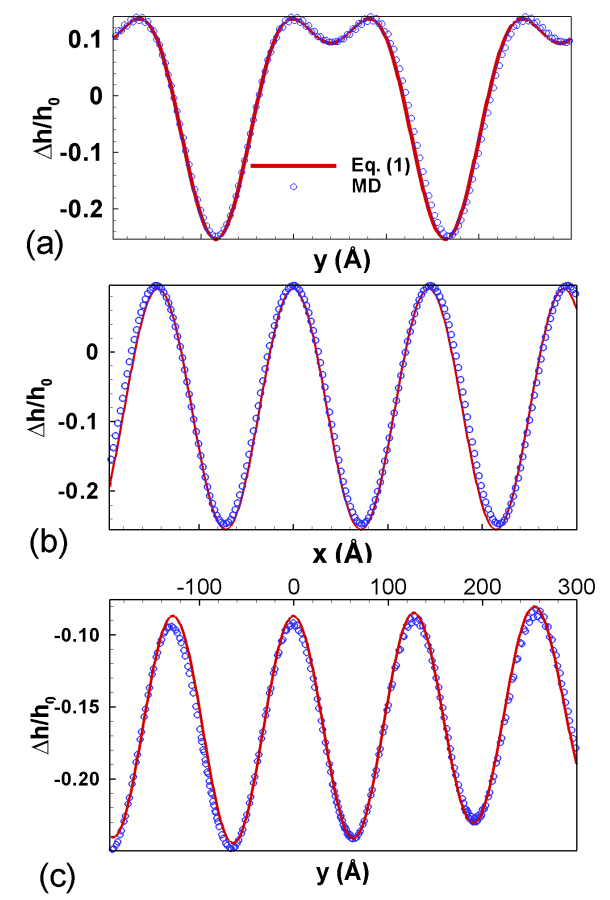

FIG. 3: (Color online) The height deformation from molecular dynamics relaxation for GE/h-BN sheet and corresponding analytical results from Eq. (1). The lattice mismatch is $\delta=1.7 \%$ and two cross sections in $(\mathrm{a}, \mathrm{b})$ correspond to $\theta=0^{\circ}$ and in (c) $\theta=0.5^{\circ}$ (circular symbols were taken from Figs. 1 $(\mathrm{a} 1, \mathrm{c} 1))$. give a more correct height scale $h_{0}$. Notice that the advantage of using MD simulation for studying effects due to MP is that it allows us to use large nit cells having nano meter size which is infeasible by ab-initio calculations. The disadvantage of using our two body potential is that it ignores any direction dependence.

In summary, we presented a general theory for the strain modulation in a graphene sheet due to the vdW interaction with a substrate having a small lattice mismatch $\delta$. The strain results in an induced pseudo magnetic field that depends strongly on the misorientation angle between GE and the substrate. The stress is distributed non-uniformly following a Moiré pattern. Our analytical theory was validated qualitatively by using atomistic simulations that we applied to the GE/BN system. This study realizes in a natural way the proposal for triaxial stress creation in GE proposed by F. Guinea et al. 9] using a h-BN sheet. The obtained Moiré pattern agrees with those found experimentally [10, 18, 20] and the induced gap agrees with recent experiments [10, 11].

Acknowledgment: This work was supported by the Flemish Science Foundation (FWO-Vl) and the Methusalem Foundation of the Flemish Government. M.N.-A was supported by the EU-Marie Curie IIF postdoc Fellowship 299855.
[1] A. K. Geim and I. V. Grigorieva, Nature (London) 499, 419 (2013).

[2] C. R Dean, A. F. Young, I. Meric, C. Lee, L. Wang, S. Sorgenfrei, K. Watanabe, T. Taniguchi, P. Kim, K. L. Shepard, and J. Hone, Nature Nanotechnol. 5, 722 (2010).

[3] W. Yang, G. Chen, Z. Shi, C-C. Liu, L. Zhang, G. Xie, M. Cheng, D. Wang, R. Yang, D. Shi, K. Watanabe, T. Taniguchi, Y. Yao, Y. Zhang, and G. Zhang, Nature Materials 12, 792 (2013).

[4] S. Tang, H. Wang, Y. Zhang, A. Li, H. Xie, X. Liu, L. Liu, T. Li, F. Huang, X. Xie, and M. Jiang, Scientific Reports 3, 2666 (2013).

[5] J. Beheshtian, A. Sadeghi, M. Neek-Amal, K. H. Michel, and F. M. Peeters, Phys. Rev. B 86, 195433 (2012).

[6] M. Yankowitz, J. Xue, D. Cormode, J. D. SanchezYamagishi, K. Watanabe, T. Taniguchi, P. JarilloHerrero, P. Jacquod, and B. J. LeRoy, Nature Phys. 8, 382 (2012).

[7] G. Giovannetti, P. A. Khomyakov, G. Brocks, P. J. Kelly, and J. van den Brink Phys. Rev. B 76, 073103 (2013); B. Sachs, T. O. Wehling, M. I. Katsnelson, and A. I. Lichetensteinn, Phys. Rev. B 84, 195414 (2011); M. Zarenia, O. Leenaerts, B. Partoens, and F. M. Peeters, Phys. Rev. B 86, 085451 (2012).

[8] M. Neek-Amal, L. Covaci, Kh. Shakouri, and F. M. Peeters Phys. Rev. B 88, 115428 (2013); M. Neek-Amal,
L. Covaci, and F. M. Peeters Phys. Rev. B 86, 041405 (2012); M. Neek-Amal and F. M. Peeters Phys. Rev. B 85, 195446 (2012).

[9] F. Guinea, M. I. Katsnelson, and A. K. Geim, Nat. Phys. 2, 31 (2010).

[10] C. R. Woods, L. Britnell, A. E. Eckman, G. L. Yu, R. V. Gorbachev, A. V. Kretinin, J. Park, L. A. Ponomarenko, M. I. Katsneslson, Yu. N. Gornostyrev, K. Watanabe, T. Tnaguchi, A. K. Geim, and K. S. Novoselov, arXiv:1401.2637 (2014).

[11] B. Hunt, J. D. Sanchez-Yamagishi, A. F. Young, M. Yankowitz, B. J. LeRoy, K. Watanabe,T. Taniguchi, P. Moon, M. Koshino, P. Jarillo-Herrero, and R. C. Ashoori. Science 340, 1427 (2013).

[12] J. Xue, J. Sanchez-Yamagishi, D. Bulmash, P. Jacquod, A. Deshpande, K. Watanabe, T. Taniguchi, P. JarilloHerrero, and Brian J. LeRoy, Nat. Mat. 10, 282 (2011).

[13] M. Neek-Amal and F. M. Peeters, Appl. Phys. Lett. 104, 041909 (2014).

[14] In fact, we performed several large scale atomistic simulations with dimensions larger than $200 \AA$ and found even for very weak vdW interaction $(10 \mathrm{meV})$ that the minimum energy configuration contains out-of-plane deformation with MP structure.

[15] J. R. Wallbank, A. A. Patel, M. Mucha-Kruczynski, A. K. Geim, and V. I. Fal'ko, Phy. Rev. B 87, 245 (2013).

[16] A. H. Castro Neto, F. Guinea, N. M. R. Peres, K. S. 
Novoselov, and A. K. Geim, Rev. Mod. Phys. 81, 109162 (2009).

[17] K. V. Zakharchenko, M. I. Katsnelson, and A. Fasolino, Phys. Rev. Lett. 102, 046808 (2009).

[18] W. Yang, G. Chen, Z. Shi, C-C. Liu, L. Zhang, G. Xie, M. Cheng, D. Wang, R. Yang, D. Shi, K. Watanabe, T. Taniguchi, Y. Yao, Y. Zhang, and G. Zhang, Nature
Materials 12, 792 (2013).

[19] P. Süle and M. Szendrö, arXiv:1402.4962 (2014).

[20] T. Mashoff, M. Pratzer, V. Geringer, T. J. Echtermeyer, M. C. Lemme, M. Liebmann, and M. Morgenstern, Nano Lett. 10, 461 (2010). 